PROCEEDINGS OF THE

AMERICAN MATHEMATICAL SOCIETY

Volume 130, Number 3, Pages 849-854

S 0002-9939(01)06101-9

Article electronically published on October 5, 2001

\title{
ON THE TOTAL CURVATURE OF CONVEX HYPERSURFACES IN HYPERBOLIC SPACES
}

\author{
ALBERT BORBÉLY
}

(Communicated by Wolfgang Ziller)

\begin{abstract}
Let $C_{1} \subset C_{2} \subset H^{n}$ be two convex compact subsets of the hyperbolic space $H^{n}$ with smooth boundary. It is shown that the total curvature of the hypersurface $\partial C_{2}$ is larger than the total curvature of $\partial C_{1}$.
\end{abstract}

\section{IntRoduction}

Let $M^{n}$ be an $n$-dimensional Riemannian manifold and let $F$ be an $(n-1)$ dimensional smooth immersed hypersurface. Denote by $A_{q}: T_{q} F \rightarrow T_{q} F$ the shape operator of $F$ at $q \in F$ with respect to a normal field defined in a neighborhood of $q$ and set $K(q)=\operatorname{det} A_{q}$. This is well defined up to sign. When $M$ is the Euclidean space, it is called the Gauss-Kronecker curvature. We adopt the same name for $K$ in general although it is no longer an intrinsic quantity of the hypersurface.

Let $H^{n}$ denote the hyperbolic space and let $C_{0} \subset C_{1}$ be two convex compact subsets with smooth boundaries. The goal of the paper is to show:

Theorem 1. With the notations introduced above we have

$$
\operatorname{Vol}\left(S^{n-1}\right) \leq \int_{\partial C_{0}} K \leq \int_{\partial C_{1}} K .
$$

Here, $K$ is computed with respect to the outward normal field of $\partial C_{i}, i=0,1$, and $\operatorname{Vol}\left(S^{n-1}\right)$ denotes the Euclidean $(n-1)$-dimensional volume of the unit sphere $S^{n-1}$.

It is well known that if $C$ is a convex compact subset of the Euclidean space, then

$$
\int_{F} K=\operatorname{Vol}\left(S^{n-1}\right)
$$

In a general Hadamard manifold $M^{n}$, as a result of the Gauss-Bonnet theorem, we have for $n=2$

$$
\operatorname{Vol}\left(S^{n-1}\right) \leq \int_{\partial C_{0}} K \leq \int_{\partial C_{1}} K
$$

Received by the editors February 15, 2000 and, in revised form, September 20, 2000.

1991 Mathematics Subject Classification. Primary 53C21.

Key words and phrases. Total curvature, Gauss-Kronecker curvature, isoperimetric inequality. This research was supported by the Kuwait University Research Grant SM 03/99. 
and for $n=3$

$$
\operatorname{Vol}\left(S^{n-1}\right) \leq \int_{\partial C_{0}} K
$$

It seems natural to wonder to what extent the above statements will hold in higher dimensions. Some partial results with respect to (2) were obtained in [1].

Although we have precise results about certain integrals on hypersurfaces due to Chern (the curvature integra [2]), the generalized Gauss-Bonnet-Chern theorem does not seem to help in higher dimension (at least not in an obvious way).

There is another motivation for trying to show that (2) is satisfied for a general nonpositively curved manifold. This is the so-called isoperimetric conjecture (see [3], 4]).

Isoperimetric Conjecture. Let $M^{n}$ be a Hadamard manifold and $D \subset M^{n}$ be a compact domain with smooth boundary. Then it satisfies the Euclidean isoperimetric inequality:

$$
\operatorname{area}(\partial D) \geq d_{n}(\operatorname{vol}(D))^{\frac{n-1}{n}},
$$

where $d_{n}=\operatorname{area}\left(S^{n-1}\right) /\left(\operatorname{vol}\left(B^{n}\right)\right)^{\frac{n-1}{n}}$.

This is now settled in dimension 4 by [3] and in dimension 3 by [4]. In fact, the main part of the proof in [4] is to show how (2) implies the isoperimetric inequality. Although it was carried out in dimension 3 only, it is very likely (and is explicitly mentioned in [4]) that it generalizes to higher dimensions. This means that a possible way of proving the isoperimetric conjecture is to establish (2) for a general Hadamard manifold.

\section{Construction of a Differential FORM}

This is a general construction due to Chern [1] which works on any Riemannian manifold $M^{n}$. Our notation follows the notation of the original paper.

Let $e_{n}$ be a unit normal field defined on some open subset of $M^{n}$. At each point extend this to an orthonormal frame $e_{1}, \ldots, e_{n}$ such that $e_{i}$ is a smooth vector field for $i=1, \ldots, n$. At least locally it is certainly possible. We now define the connection forms as

$$
\omega_{j}^{i}(X)=\left\langle\nabla_{X} e_{j}, e_{i}\right\rangle
$$

where $\langle.,$.$\rangle denotes the metric on M^{n}$ and $X$ is a vector field. The curvature form is defined as

$$
\Omega_{j}^{i}(X, Y)=-\left\langle R(X, Y) e_{j}, e_{i}\right\rangle,
$$

where $R(X, Y)$ denotes the curvature tensor defined as: $R(X, Y) Z=\nabla_{Y} \nabla_{X} Z-$ $\nabla_{X} \nabla_{Y} Z+\nabla_{[X, Y]} Z$. Then Cartan's second structural equation reads as

$$
\Omega_{j}^{i}=d \omega_{j}^{i}+\omega_{k}^{i} \omega_{j}^{k}
$$

where we use the usual summation convention, summing over repeated indices.

The differential form which is of interest to us is defined as:

$$
\Phi=\omega_{n}^{1} \omega_{n}^{2} \ldots \omega_{n}^{n-1} .
$$

This is an $(n-1)$-form on $M^{n}$ which is essentially the same as Chern's form denoted by $\Phi_{0}$ in [2]. More precisely, $\Phi_{0}$ is an $(n-1)$-form on the unit tangent bundle and 
$\Phi$ is its pull back via the map $E_{n}: M^{n} \rightarrow T M^{n}$ defined as $E_{n}(p)=e_{n}(p)$. As a consequence we have the following important observation:

Fact. The differential form $\Phi$ depends only on the vector field $e_{n}$. It does not depend on how $e_{n}$ is extended to an orthonormal frame $e_{1}, \ldots, e_{n}$.

From the second structural equation (4) one can derive that

$$
d \Phi=\sum \epsilon_{i_{1} \ldots i_{n-1}} \Omega_{n}^{i_{1}} \omega_{n}^{i_{2}} \ldots \omega_{n}^{i_{n-1}},
$$

where $\epsilon_{i_{1} \ldots i_{n-1}}$ is the Kronecker index which is equal to +1 or -1 according to whether the permutation $i_{1} \ldots i_{n-1}$ of the numbers $1,2, \ldots, n-1$ is even or odd and the summation is extended over all the indices $i_{1} \ldots i_{n-1}$ subject to the condition $i_{2}<i_{3}<\ldots<i_{n-1}$. It is essentially the same as the $n$-form $\Psi_{0}$ in [2].

\section{Convex exhaustion}

The other important ingredient is a lemma about convex exhaustion. Although we only need this in the hyperbolic space we state it for Hadamard manifolds. We say that a convex set $C$ with smooth boundary is strictly convex if the second fundamental form of the boundary (with respect to the outward normal) is positive definite everywhere.

Lemma 1. Let $M^{n}$ be a Hadamard manifold and $C_{0} \subset \operatorname{int}\left(C_{1}\right)$ be two compact strictly convex subsets with smooth boundary. Then there exists a continuous function $F: C_{1}-\operatorname{int}\left(C_{0}\right) \rightarrow[0,1]$ which is smooth in the interior such that: $\nabla F \neq 0, \partial C_{i}=\left\{p \in C_{2}-\operatorname{int}\left(C_{1}\right): F(p)=i\right\}$ for $i=0,1$ and the sublevel sets $C_{b}=\left\{p \in C_{2}-\operatorname{int}\left(C_{1}\right): F(p) \leq b\right\}$ for $0 \leq b \leq 1$ are convex.

Proof. The statement is clear intuitively. Denote by $\varrho_{i}$ the distance function from the set $\partial C_{i}$ for $i=0,1$.

For $\delta>0$ we set $N_{2 \delta}=\left\{p \in C_{1}: \varrho_{1}(p)<2 \delta\right\}$. Since $\partial C_{1}$ has a positive definite second fundamental form, we can choose $\delta>0$ small enough such that: $3 \delta<\operatorname{dist}\left(C_{0}, \partial C_{1}\right)$, the function $\varrho_{1}$ is smooth on $N_{2 \delta}$ (there are no focal points of $\partial C_{1}$ inside $\left.N_{2 \delta}\right)$ and

$$
-D^{2} \varrho_{1}>c_{1}>0
$$

when restricted to $\nabla \varrho_{1}^{\perp}$ for some positive constant $c_{1}$. Here, $D^{2} \varrho_{1}$ denotes the Hessian and we adapted the notation that $-D^{2} \varrho_{1}>c_{1}$ on $\nabla \varrho_{1}^{\perp}$ if $-D^{2} \varrho_{1}(X, X)>$ $c_{1}$ for every unit tangent vector $X \in \nabla \varrho_{1}^{\perp}$. We observe also that on $N_{2 \delta}$ the following inequality holds for the angle between the gradients:

$$
\measuredangle\left(\nabla \varrho_{0},-\nabla \varrho_{1}\right)<\pi / 2-\alpha,
$$

for some $\alpha>0$ depending on $\delta$.

We are going to construct $F$ in the form

$$
F=1-e^{-a \varrho_{0}} f^{\epsilon} .
$$

Here $f=h\left(\varrho_{1}\right)$ is the reparametrized distance function from $\partial C_{1}$ and $h$ is a fixed smooth increasing real function $h:[0, \infty) \rightarrow[0,1]$ such that $h(t)=\frac{1}{2 \delta} t$ for $0 \leq t \leq \delta$ and $h(t)=1$ on $[2 \delta, \infty)$. For the derivative and the Hessian of $f$ we have

$$
d f=h^{\prime} d \varrho_{1} \quad \text { and } \quad D^{2} f=h^{\prime \prime} d \varrho_{1} \otimes \varrho_{1}+h^{\prime} D^{2} \varrho_{1} .
$$

The choice of $a, \epsilon$ will be discussed later. 
First, we show that the derivative of $F$ is never zero. From the definition we obtain

$$
d F=e^{-a \varrho_{0}} f^{\epsilon}\left(a d \varrho_{0}-\frac{\epsilon}{f} d f\right) .
$$

Then, taking into consideration that $\nabla f \| \nabla \varrho_{1}$, the statement follows from (7).

To show that the sublevel sets are convex we need to show that the Hessian $D^{2} F$ is positive definite on $\nabla F^{\perp}$. We have

$$
\begin{aligned}
D^{2} F & =e^{-a \varrho_{0}}\left(-a^{2} d \varrho_{0} \otimes d \varrho_{0}+a D^{2} \varrho_{0}\right) f^{\epsilon} \\
& +\epsilon e^{-a \varrho_{0}} f^{\epsilon-1}\left(\frac{1-\epsilon}{f} d f \otimes d f-D^{2} f\right) \\
& +\epsilon a e^{-a \varrho_{0}} f^{\epsilon-1}\left(d \varrho_{0} \otimes d f+d f \otimes d \varrho_{0}\right) .
\end{aligned}
$$

The argument depends on certain estimates of the Hessians $D^{2} \varrho_{i}$ for $i=0,1$, on various subspaces. It will be useful to keep in mind that $\varrho_{i}$ (for $i=0,1$ ) are distance functions; therefore the gradients $\nabla \varrho_{i}$ are eigenvectors of $D^{2} \varrho_{i}$ with eigenvalue zero.

First, we consider the region where $f \equiv 1$. On this region $d f, D^{2} f=0$ and $\nabla F \| \nabla d \varrho_{0}$. Therefore $D^{2} F=a f^{\epsilon} e^{-a \varrho_{0}} D^{2} \varrho_{0}$, when it is restricted to vectors in $\nabla F^{\perp}$. But on this subspace $D^{2} \varrho_{0}$ is positive definite since it is the distance function from a convex set in a Hadamard manifold.

Next, we consider the region where $1 / 2<f<1$. Since $C_{0}$ is strictly convex and compact, we know that $D^{2} \varrho_{0}>c_{2}>0$ for some $c_{2}>0$, when restricted to $\left(\nabla \varrho_{0}\right)^{\perp}$. Therefore, taking (6) and (9) into account, we conclude that $D^{2} \varrho_{0}>c_{3}>0$ for some positive constant $c_{3}$, when restricted to $\nabla F^{\perp}$. So, for a small enough $a$, where the choice of $a$ depends only on $c_{3}$, the term $a D^{2} \varrho_{0}$ will dominate $a^{2} d \varrho_{0} \otimes d \varrho_{0}$. This will remain true throughout the whole region $C_{1}-C_{0}$. The derivatives of $h$ are bounded and so are the terms $d f$ and $D^{2} f$. Therefore, for a small enough $\epsilon$, the term $a D^{2} \varrho_{0}$ will dominate all the other terms as well. The choice of $\epsilon$ depends on $a$ and on the bounds for $d f$ and $D^{2} f$.

At last, we consider the region where $0<f<1 / 2$. It is clear from the definition of the function $f$ that this region is a subset of $N_{2 \delta}$. As before, $a D^{2} \varrho_{0}$ will dominate $a^{2} d \varrho_{0} \otimes d \varrho_{0}$. The term involving $d f \otimes d f$ is positive semi-definite. As for the rest of the terms, we will show that the term involving $-D^{2} f$ will dominate, for a small enough $a$, the term involving $d \varrho_{0} \otimes d f+d f \otimes d \varrho_{0}$. Since $h$ is linear on this region, from (6) we obtain $-D^{2} f=-h^{\prime} D^{2} \varrho_{1}>c_{1} / 2 \delta>0$, when restricted to $\left(\nabla \varrho_{1}\right)^{\perp}$. Taking into consideration ( 7 ) and (9) we conclude that $-D^{2} f>c_{4}>0$ for some sufficiently small $c_{4}>0$, when restricted to $\nabla F^{\perp}$. The constant $c_{4}$ depends on $c_{1}, \delta$ and the angle $\alpha$. So, if the constant $a>0$ was chosen small enough, where the choice of $a$ depended only on $c_{2}$ and $c_{4}$, then $-D^{2} f$ dominates the term involving $d \varrho_{0} \otimes d f+d f \otimes d \varrho_{0}$. This concludes the proof of the lemma.

\section{Proof of Theorem 1}

With the preparation done in the previous sections, the proof of the theorem is simple. Let us return to the hyperbolic space $H^{n}$.

First, we prove the inequality between the two integrals. Assume that $C_{0} \subset$ $\operatorname{int}\left(C_{1}\right)$ and both sets are strictly convex. We are going to show that

$$
\int_{\partial C_{0}} K \leq \int_{\partial C_{1}} K
$$


The general case will follow by a trivial limiting procedure.

Let $F$ be the smooth function of Lemma 1 and define the unit vector field $e_{n}$ by $e_{n}=\nabla F /|\nabla F|$. This is defined on $\operatorname{int}\left(C_{1}\right)-C_{0}$ but it extends continuously to the boundary. To the vector field $e_{n}$ we construct the form $\Phi$ as in the previous section and by Stokes's theorem we have

$$
\int_{\partial C_{0} \cup \partial C_{1}} \Phi=\int_{C_{1}-C_{0}} d \Phi
$$

To evaluate the integrals in (12) we are going to compute the forms $\Phi$ and $d \Phi$. Let $q \in C_{1}-\operatorname{int}\left(C_{0}\right)$ be an arbitrarily chosen point.

Since $\Phi$ depends only on the vector field $e_{n}$, we can express $\Phi$ in a special frame. Let us choose the frame $e_{1}, \ldots, e_{n}$ such that at the point $q \in C_{1}-\operatorname{int}\left(C_{0}\right)$ the vectors $e_{1}, \ldots, e_{n-1}$ are the principal directions for the hypersurface $\{F=F(q)\}$. For the other points of the hypersurface the vectors $e_{1}, \ldots, e_{n-1}$ may no longer be principal directions. Then, from the definition of the $\omega_{n}^{i}$ 's we have

$$
\omega_{n}^{i}\left(e_{j}\right)=\delta_{j}^{i} \lambda_{j}, \quad \text { for } \quad 1 \leq i, j \leq n-1
$$

at $q \in C_{1}-\operatorname{int}\left(C_{0}\right)$, where $\lambda_{j}$ denotes the principal curvature at $q$ of the hypersurface $\{F=F(q)\}$ in the direction of $e_{j}$ and $\delta_{j}^{i}$ is the Kronecker symbol. Therefore

$$
\Phi\left(e_{1}, \ldots, e_{n-1}\right)=\lambda_{1} \cdot \ldots \cdot \lambda_{n-1}=K
$$

at $q \in C_{1}-\operatorname{int}\left(C_{0}\right)$, where $K$ denotes the Gauss-Kronecker curvature of the hypersurface $\{F=F(q)\}$ with respect to the normal field $e_{n}$. Since $q \in C_{1}-\operatorname{int}\left(C_{0}\right)$ was chosen arbitrarily, the left-hand side of (12) reads as follows:

$$
\int_{\partial C_{0} \cup \partial C_{1}} \Phi=\epsilon_{n 12 \ldots n-1}\left(\int_{\partial C_{1}} K-\int_{\partial C_{0}} K\right)=(-1)^{n-1}\left(\int_{\partial C_{1}} K-\int_{\partial C_{0}} K\right) .
$$

The curvature tensor has the form $R(X, Y) Z=\langle Y, Z\rangle X-\langle X, Z\rangle Y$; therefore

$$
\Omega_{n}^{i}\left(e_{j}, e_{k}\right)=0
$$

if $\{j, k\} \neq\{i, n\}$ as sets. From this and (13) we have

$$
\Omega_{n}^{i_{1}} \omega_{n}^{i_{2}} \ldots \omega_{n}^{i_{n-1}}\left(e_{1}, \ldots, e_{n}\right)=\epsilon_{i_{1} n i_{2} \ldots i_{n-1}} K_{i_{1} n} \lambda_{i_{2}} \cdot \ldots \cdot \lambda_{i_{n-1}}
$$

at $q \in C_{1}-\operatorname{int}\left(C_{0}\right)$, where the indices satisfy the condition $i_{2}<i_{3}<\ldots<i_{n-1}$ and $K_{\text {in }} \equiv-1$ denotes the sectional curvature of the two-plane determined by $e_{i}, e_{n}$ and $\lambda_{i}$ is the principal curvature at the point $q \in C_{1}-\operatorname{int}\left(C_{0}\right)$ in the direction of $e_{i}$. Then (12) reads as follows:

$$
\int_{\partial C_{1}} K-\int_{\partial C_{0}} K=\int_{C_{1}-C_{0}} \sum-K_{i_{1} n} \lambda_{i_{2}} \cdot \ldots \cdot \lambda_{i_{n-1}},
$$

where the summation is extended over all the indices $i_{1} \ldots i_{n-1}$ subject to the condition $i_{2}<i_{3}<\ldots<i_{n-1}$.

Since all sublevel sets are convex, all the principal curvatures are positive. Therefore, the integral on the right-hand side is positive. This completes the proof of the theorem, when $C_{0} \subset \operatorname{int}\left(C_{1}\right)$ and both sets are strictly convex. The general case follows by slightly "blowing up" the sets; that is, instead of $C_{0}$ we consider an $\eta$-neighborhood $C_{\eta}$ and instead of $C_{1}$ we take a $2 \eta$-neighborhood $C_{1+2 \eta}$. These are now strictly convex sets satisfying the conditions set forth at the beginning of the proof. Then letting $\eta$ go to 0 will yield the general case. 
All that remains is to prove the inequality

$$
\operatorname{Vol}\left(S^{n-1}\right) \leq \int_{\partial C_{0}} K
$$

This is a simple consequence of (11). Choose a ball $B_{\epsilon}$ inside $C_{0}$. Applying (11), we obtain

$$
\int_{\partial B_{\epsilon}} K<\int_{\partial C_{0}} K
$$

Letting $\epsilon$ go to 0 will yield the desired inequality. This completes the proof of the theorem.

\section{REFERENCES}

1. Borbély, A., On the total curvature of hypersurfaces in negatively curved manifold, preprint.

2. Chern, S-S., On the curvatura integra in a Riemannian manifold, Annals of Math. 46 (1945), 674-684. MR 7:328c

3. Croke, C., A sharp four dimensional isoperimetric inequality, Comment. Math. Helv. 59 (1984), 187-192. MR 85f:53060

4. Kleiner, B., An isoperimetric comparison theorem, Invent. Math. 108 (1992), 37-47. MR 92m:53056

Department of Mathematics and Computer Science, Kuwait University, P.O. Box 5969, SAFAT 13060, KuWAit

E-mail address: borbely@mcs.sci.kuniv.edu.kw 